\title{
Riscos Psicossociais Relacionados ao Trabalho: Perspectivas Teóricas e Conceituais
}

\author{
Pedro Augusto Crocce Carlotto ${ }^{1}$, Roberto Moraes $\mathrm{Cruz}^{2}$, Romilda Guilland ${ }^{3}$, Ricelli Endrigo \\ Ruppel $^{4}$ da Rocha, Patrícia Dalagasperina ${ }^{5}$, Daniela Ornellas Ariño ${ }^{6}$
}

\author{
Recibido: Aceptado: \\ DOI: $10.21772 /$ ripo.v37n1a04
}

\begin{abstract}
Resumo
Riscos psicossociais no trabalho são discutidos sob diferentes nomenclaturas, tais como como fatores psicossociais, fatores de riscos psicossociais, estressores e aspectos psicossociais. O objetivo desta revisão é analisar criticamente os aspectos teóricos e conceituais que sustentam as diferentes denominações para riscos psicossociais relacionados ao trabalho. Foram selecionados 64 artigos para composição desta revisão, publicados entre os anos de 2006-2016, nas bases Scopus e Web of Science. Em função dos termos serem descritos indistintamente na literatura, não há uma clara definição de quais fatores são considerados riscos psicossociais ocupacionais. Este cenário afeta a maneira como se compreendem os riscos psicossociais no trabalho, tornando-os de difícil definição e avaliação. O construto riscos psicossociais ainda é um conceito ambíguo e precisa ser melhor operacionalizado em suas facetas e na legislação em Saúde e Segurança do Trabalho. Salienta-se a necessidade de um aperfeiçoamento do conceito de riscos psicossociais ocupacionais, pouco explorado na literatura.
\end{abstract}

Palavras chave: Riscos psicossociais no trabalho, Fatores psicossociais. Riscos psicossociais ocupacionais, Saúde e Segurança no Trabalho.

\section{Work-Related Psychosocial Risks theoretical and conceptual perspectives}

\begin{abstract}
Psychosocial risks at work are discussed under different terminologies - psychosocial factors, psychosocial risk factors, psychosocial stressors and aspects. The objective of this review is to critically analyze the theoretical and conceptual aspects that support the different denominations for psychosocial risks. For this review, 64 publications were selected, published from the years of 2006 to 2016, on Scopus and Web of Science databases. Since psychosocial risks are described by different terms, indiscriminately, there is a lack of definition about which factors are actually considered occupational psychosocial risks. This scenario affects how psychosocial risks at work are understood, making it harder to define and measure them. Psychosocial risks at work require more theoretical and operational definitions, and more support from Occupational Safety and Health laws. The construct of psychosocial risks is still an ambiguous concept and needs to be better operationalized in its facets and legislation on Occupational Health and Safety. It is necessary to improve the concept of occupational psychosocial risks, little explored in the literature.
\end{abstract}

Keywords: Work-Related Psychosocial Risks, Psychosocial factors at work, Occupational Psychosocial Risks, Psychosocial hazards. Safety and Health at Work.

\footnotetext{
1 M.S. en psicología. Laboratório Fator Humano - Departamento de Psicologia- Centro de Filosofia e Ciências Humanas (CFH) - Campus universitário, s/n - Trindade, Florianópolis - SC, 88040-900, Brasil. E-mail: pedroaccarlotto@gmail.com

2 Ph.D. en Psicología Laboratório Fator Humano - Departamento de Psicologia- Centro de Filosofia e Ciências Humanas (CFH) - Campus universitário, s/n - Trindade, Florianópolis - SC, 88040-900, Brasil. E-mail: robertocruzdr@gmail.com

3 Ph. D. en Psicología. Laboratório Fator Humano - Departamento de Psicologia- Centro de Filosofia e Ciências Humanas (CFH) - Campus universitário, s/n - Trindade, Florianópoli. E-mail: guil.ro@hotmail.com

4 Doctor en Educación Física. Universidade Alto Vale do Rio do Peixe, UNIARP, Brasil. E-mail: ricelliendrigo@yahoo.com.br

5 Doctora en Psicología Laboratório Fator Humano - Programa de Pós-Graduação em Psicologia. E-mail: pati-d@hotmail.com

6 Doctorando Psicología. Laboratório Fator Humano - Programa de Pós-Graduação em Psicologia. E-mail: daniela.arino@gmail.com
}

Note: part of this paper was presented at the XV Caribbean Congress of Psychology, April 7 - 8, 2018 at the Universidad Tecnológica de Santiago (UTESA), Puerto Plata, Dominican Republic.

Cómo citar este artículo: Crocce Carlotto, P. A.; Moraes Cruz, R. \& Guilland, R. (2018). Riscos Psicossociais Relacionados ao Trabalho: Perspectivas Teóricas e Conceituais. Revista Interamericana de Psicología Ocupacional, 37(1), 52-70. DOI: 10.21772/ripo.v37n1a04 


\section{Introdução}

Riscos psicossociais relacionados ao trabalho são aspectos do desenho do trabalho, do gerenciamento e do contexto organizacional, com potencial de causar danos à saúde e segurança no trabalho (International Labour Organization, 2017; Stavroula Leka \& Cox, 2008; Weissbrodt \& Giauque, 2017). Estão altamente associados ao estresse, às doenças físicas e mentais relacionadas ao trabalho e aos problemas relativos ao desempenho (International Labour Organization, 2017; Organización Internacional del Trabajo, 2010, 2013).

Desde a década de 1980, há vasta quantidade de estudos acerca de riscos psicossociais relacionados ao trabalho e, apesar de sua crescente relevância e preocupação, face aos impactos que produzem nas organizações, há aspectos teóricos e conceituais que merecem ser objetos de reflexão crítica. Um dos principais aspectos, nesse sentido, é o uso de diferentes terminologias que definem riscos psicossociais (Kop, Althaus, Formet-Robert, \& Grosjean, 2016; Weissbrodt \& Giauque, 2017).

Em 1984, a Organização Internacional do Trabalho (OIT) definiu que "fatores psicossociais" são o resultado da interação entre aspectos do ambiente organizacional e do indivíduo, com influências na saúde e desempenho dos trabalhadores (International Labour Organization, 1984), nomenclatura ainda utilizada na atualidade (Chirico, 2016; Farnacio, Pratt, Marshall, \& Graber, 2017). Observa-se, desde então, o uso também dos termos "fatores de risco psicossociais" (Madsen et al., 2017; Organización Internacional del Trabajo, 2013) e riscos psicossociais (Stravoula Leka, Jain, Iavicoli, \& Di Tecco, 2015), sendo este último utilizado principalmente por agências e órgãos de saúde ocupacional influenciados pelo contexto europeu, que se têm se destacado pelos avanços em programas de gerenciamento desse tipo de risco (Weissbrodt \& Giauque, 2017). Há, ainda, terminologias como "aspectos psicossociais"
(Gray, Adefolarin, \& Howe, 2011; International Labour Organization, 1984), ou "estressores psicossociais" relacionados ao trabalho (Chirico, 2017).

$\mathrm{Na}$ literatura há pouca clareza sobre quais elementos constituem riscos psicossociais ocupacionais (Chirico, 2017; Kop et al., 2016). Esta revisão sistemática foi realizada no intuito de refletir criticamente acerca das diferenças conceituais os termos utilizados para definir riscos psicossociais no trabalho, bem como relacionálos às diferentes perspectivas teóricas que os sustentam.

\section{Método}

Esta revisão sistemática utilizou os procedimentos metodológicos estabelecidos pelo guia Preferred Reporting Items for Systematic Reviews and Meta-Analyses - PRISMA (Moher, Liberati, Tetzlaff, Altman, \& Group, 2009). Foram consultadas as bases de dados Web of Science ${ }^{\circledR}$ (Thomson Reuters) e Scopus ${ }^{\circledR}$ (Elsevier), ambas consideradas bases com o maior alcance de publicações científicas na atualidade, bem como alto fator de impacto (Aghaei Chadegani et al., 2013). Optou-se por investigar os conceitos referidos por meio dos quais são encontrados no Medical Subject Headings (MeSH) e no sistema de thesaurus da American Psychological Association (APA), além de outros descritores que são utilizados nas publicações especializadas: psychosocial hazards, psychosocial factors, psychosocial risks, psychosocial stressors e psychosocial aspects (Chirico, 2017; International Labour Organization, 2017; Jacukowicz, 2016; Rick, Briner, Daniels, Perryman, \& Guppy, 2001). Os termos encontrados foram testados individualmente e, por fim, chegouse à combinação: "Psychosocial risk*" OR "psychosocial factor*" OR "psychosocial aspect*" OR "psychosocial hazard*" OR "psychosocial stressor*" AND Job OR occupational OR work* AND Review OR overview OR "systematic review" OR "integrative review" AND Theor* OR model* OR concept* OR definition*. O 
período de busca dos artigos foi de janeiro de 2006 a dezembro de 2017.

Para a inclusão dos artigos, foram estabelecidos os seguintes critérios: a) artigos teóricos e de revisões nos idiomas português, espanhol e inglês; b) com definição clara de embasamento teórico de riscos psicossociais no trabalho. Os critérios de exclusão dos artigos foram: a) artigos empíricos; b) estudos sobre riscos psicossociais não relacionados ao trabalho e ou ao ambiente ocupacional. Inicialmente, os pesquisadores foram separados em duplas e foi determinado uma combinação de descritores para cada uma delas. Cada dupla realizou de maneira independente cada etapa do processo de revisão. Divergências no processo de inclusão e exclusão foram decididas em uma reunião de consenso entre os pesquisadores. Os resultados da busca são detalhados na Figura 1.

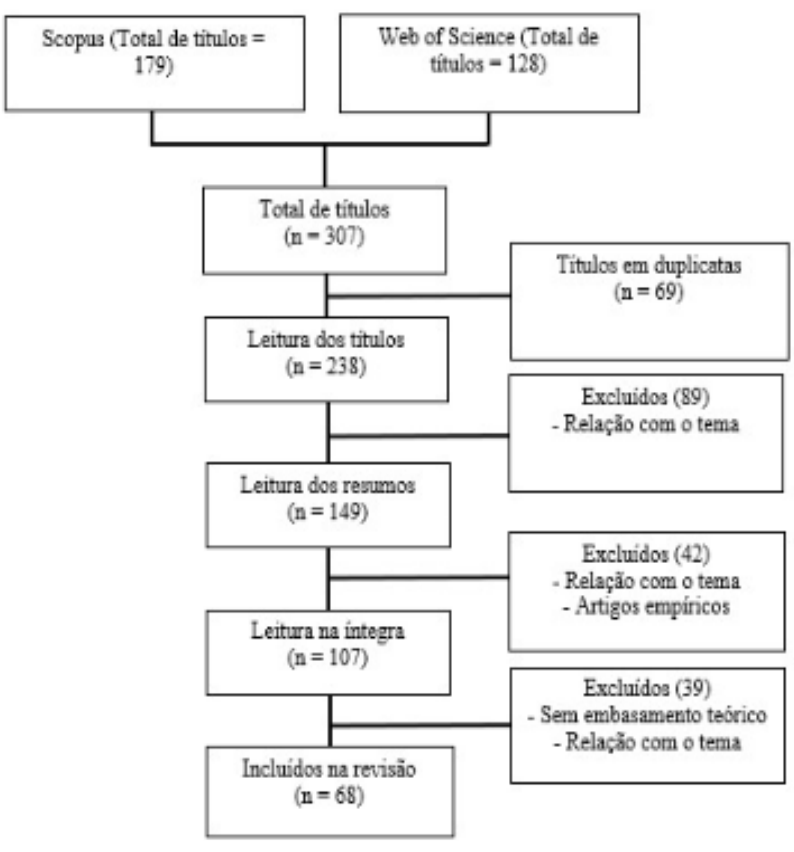

Figura 1: Fluxograma de identificação e seleção dos artigos para revisão sistemática sobre riscos e fatores de risco psicossociais no trabalho
Foram identificados 307 estudos relevantes para a revisão integrativa sobre os pressupostos teóricos e conceituais de riscos psicossociais relacionados ao trabalho. Após a exclusão dos artigos duplicados entre as bases de dados, leitura dos títulos e resumos e a leitura na íntegra, 68 artigos $(22,5 \%)$ contemplaram os critérios de inclusão para o estudo (Figura 1). Foram coletados os termos descritores referidos em cada artigo, bem como os modelos teóricos referidos. Os termos e modelos revisados foram identificados por meio da leitura de cada artigo na íntegra, com o auxílio do software Mendeley. Alguns artigos traziam mais de um termo ou modelo, e estes também foram contabilizados. Foi feito um cruzamento entre termos e modelos teóricos, com auxílio dos softwares Microsoft Excel e Access (versões 2016), conforme exposto na Tabela 1.

\section{Resultados}

Os diferentes modelos teóricos foram sistematizados em três categorias: teorias clássicas do estresse, modelos teóricos emergentes e modelos de gestão de riscos ocupacionais. Na categoria de teorias clássicas do estresse, foram alocados os modelos teóricos que investigam riscos psicossociais no trabalho sob a ótica de estressores, embasados nas teorias de Selye, Lazarus e também do job strain, desenvolvido por Robert Karasek e associado ao modelo DemandaControle e Apoio Social. Também foi incluído nesta categoria o modelo teórico Effort-Reward Imbalance (ERI), proposto por Siegrist.

$\mathrm{Na}$ categoria modelos emergentes, foram alocados os modelos surgidos a partir dos anos 2000 para a compreensão de riscos psicossociais no trabalho, como o modelo psicossocial de Copenhagen e o modelo de Justiça Organizacional. Na categoria modelos de gestão de saúde ocupacional, foram alocados os modelos teóricos perspectivados sob a ótica da Ergonomia e dos trabalhos de programas de gerenciamento de riscos psicossociais no trabalho, proposto por comitês e agências de saúde ocupacional, tendo 
Tabela 1. Relação entre modelos teóricos e termos empregado no escopo dos riscos psicossociais no trabalho

\begin{tabular}{|c|c|c|}
\hline Modelos & Conceitos empregados & \\
\hline \multirow{19}{*}{$\begin{array}{l}\text { Teorias clássicas do estresse } \\
\text { e job strain }\end{array}$} & Aspects of psychosocial work environment & Psychosocial protective factor \\
\hline & Characteristics of psychosocial work environment & Psychosocial risk factors \\
\hline & Job characteristics & Psychosocial risks \\
\hline & Job demands & Psychosocial stress \\
\hline & Job stressors & Psychosocial stressors \\
\hline & Occupational hazards & Psychosocial variables \\
\hline & Organizational constraints & Psychosocial work characteristics \\
\hline & Organizational factors & Psychosocial work conditions \\
\hline & Organizational work factors & Psychosocial workplace conditions \\
\hline & Psychological and social factors & Psychosocial workplace factors \\
\hline & Psychological risk factors & Risk factors \\
\hline & Psychosocial and organizational work factors & Stressful work environment \\
\hline & Psychosocial aspects & Stressors \\
\hline & Psychosocial demands & Work stressors \\
\hline & Psychosocial factors & Working or employment conditions \\
\hline & Psychosocial hazards & Work-related environmental factors \\
\hline & Psychosocial job demands & Work-related psychosocial factors \\
\hline & Psychosocial job factors & $\begin{array}{l}\text { Work-related psychosocial risk } \\
\text { factors }\end{array}$ \\
\hline & Psychosocial job stressors & Work-related psychosocial stressors \\
\hline \multirow{6}{*}{ Modelos emergentes } & Job stressors & Psychosocial risk factors \\
\hline & Organizational constraints & Psychosocial risks \\
\hline & Organizational work factors & Psychosocial work environment \\
\hline & Psychological and social factors & Psychosocial work factors \\
\hline & Psychosocial and organizational work factors & Psychosocial work stressors \\
\hline & Psychosocial factors & Work-related psychosocial factors \\
\hline \multirow{14}{*}{$\begin{array}{l}\text { Modelos de gestão de riscos } \\
\text { ocupacionais }\end{array}$} & Aspects of psychosocial work environment & Psychosocial occupational exposures \\
\hline & Characteristics of psychosocial work environment & Psychosocial risks \\
\hline & Job stressors & Psychosocial risk factors \\
\hline & Occupational hazards & Psychosocial stressors \\
\hline & Occupational psychosocial factors & Psychosocial variables \\
\hline & $\begin{array}{l}\text { Organizational and psychosocial contexts (influencing } \\
\text { factors) }\end{array}$ & Psychosocial work environment \\
\hline & Organizational factors & $\begin{array}{l}\text { Psychosocial work environment } \\
\text { factors }\end{array}$ \\
\hline & Psychosocial and organizational conditions & Psychosocial work factors \\
\hline & Psychosocial aspects & Psychosocial working environment \\
\hline & Psychosocial demands & Stressful work environment \\
\hline & Psychosocial factors & Stressors \\
\hline & Psychosocial factors at work & Work stressors \\
\hline & Psychosocial hazards & Working or employment conditions \\
\hline & Psychosocial job factors & Work-related psychosocial factors \\
\hline
\end{tabular}

Nota: Elaboração dos autores (2018). 
como expoente o Psychosocial Risk Management European Framework (PRIMA-EF), proposto por Stavroula Leka e Tom Cox. Os modelos são discutidos em relação aos principais termos que utilizam e seus fundamentos teóricos. Em seguida, são problematizados os diferentes graus de precisão e abrangência dos termos e conceitos utilizados, muitas vezes usados como sinônimos na literatura especializada.

\section{Discussão}

Perspectivas teóricas a respeito do estresse estudam como o organismo reage a eventos que perturbam sua homeostase. Tais eventos se originam também de estressores do ambiente psicossocial do organismo, não apenas sendo agentes físicos (Katsarou, Triposkiadis, \& Panagiotakos, 2013). Esta perspectiva teórica, iniciada por Walter Cannon na década de 1930, e aprofundada por Hans Selye na década de 1950, foi incorporada aos estudos em gestão de pessoas e saúde ocupacional, visando entender melhor o comportamento dos trabalhadores (Ganster \& Rosen, 2013; Vischer, 2007).

A ênfase em como o organismo percebe tais estressores, e o modo como o faz, foi trazida posteriormente pelos estudos de Lazarus, a partir da década de 1970 (Laisné, Lecomte, \& Corbière, 2012). O organismo, até então considerado passivo quando exposto a estressores do ambiente, passa a ser considerado como dotado de papel ativo na percepção dos estressores, com influência de processos cognitivos na apreensão e interação com aspectos do ambiente. É a partir desta percepção do ambiente pelo organismo que determinados aspectos seriam considerados, portanto, estressores (Katsarou et al., 2013; Laisné et al., 2012; Vischer, 2007). Uma das grandes contribuições de Lazarus foi a tentativa de caracterizar que o estresse resulta da interação entre indivíduo e ambiente (Ganster \& Rosen, 2013). Esta perspectiva encontra similaridade no conceito de "fatores psicossociais do trabalho", na qual os agentes do ambiente psicossocial de trabalho, por si só, não têm potencial para causar provocar estresse, mas quando associado às características do trabalhador, e este produto é denominada de fator. Fator de risco psicossocial ganha relevância na década de 1980 , contemporâneo aos estudos de Lazarus, e ainda hoje é bastante utilizado (Farnacio et al., 2017; Villalobos, Vargas, Rondón, \& Felknor, 2013), principalmente em pesquisas sobre estresse no trabalho (Chida \& Steptoe, 2009; Eller et al., 2009; D. S. Kim \& Kang, 2010; Kivimäki et al., 2006; Thayer, Yamamoto, \& Brosschot, 2010).

Posteriormente, surge o Modelo Alostático (Allostatic Model Load - AL), baseado na compreensão do processo cognitivo como preponderante na percepção do estresse. Processos alostáticos referem-se aos efeitos biológicos no organismo decorrentes da exposição crônica ao estresse (Ganster \& Rosen, 2013; Ilies, Aw, \& Lim, 2016; Schnall, Dobson, \& Landsbergis, 2016). Contudo, o modelo teórico mais destacado e proeminente na literatura a respeito de riscos psicossociais no trabalho, baseados nas teorias do estresse, é o modelo job strain (Gerin \& James, 2010; Kivimäki et al., 2006; Kraatz, Lang, Kraus, Münster, \& Ochsmann, 2013; Madsen et al., 2017; Szerencsi et al., 2012). Strain é termo definido como força que puxa ou estica algo a um nível extremo e danificador, magnitude de uma deformação ou demanda excessiva sobre a força, recursos ou capacidades de algo ou alguém (Oxford University Press, 2018). Este modelo trabalha com a relação entre exigências do ambiente e a capacidade de manejo, controle e enfrentamento do indivíduo. É tão associado ao contexto do trabalho que, termos genéricos da teoria do estresse, como "organismo" e "ambiente", são substituídos por termos como "trabalhador" e "exigências laborais" (Eller et al., 2009; Gerin \& James, 2010; Kivimäki et al., 2006; Schnall et al., 2016).

O modelo Demanda-Controle, proposto por Karasek (1979), é referido como sendo o modelo teórico que mais se aproxima da teoria de job strain e melhor explica seus pressupostos 
(Siegrist, 2011; Szerencsi et al., 2012). O principal mérito da teoria é retomar conceitos da teoria de job strain altos níveis de estresse (high strain) com alta demanda e baixo controle, bem como com o apoio social percebido pelo trabalhador (Bongers, Ijmker, Van Den Heuvel, \& Blatter, 2006; Eller et al., 2009; Kristman et al., 2016), sendo esta terceira dimensão incorporada posteriormente ao modelo teórico (Siegrist, 2011). O modelo Demanda-Controle e Apoio Social é um dos mais citados na literatura (Bonneterre, Liaudy, Chatellier, Lang, \& De Gaudemaris, 2008; Eller et al., 2009), e ao qual é atribuído maior poder explicativo (Chirico, 2016; Ganster \& Rosen, 2013; Jacukowicz, 2016).

Este modelo é visto como tendo caráter mais nomotético do que a perspectiva transacional do estresse de Lazarus, que possui foco no indivíduo (Skogstad, Torsheim, Einarsen, \& Hauge, 2011). Tanto o é que o modelo Demanda, Controle e Apoio Social (ou simplesmente job strain) é um dos mais utilizados em investigações epidemiológicas, que visam a associar a exposição a fatores psicossociais com agravos à saúde. Incluem-se nesses agravos, principalmente, doenças musculoesqueléticas (Bongers et al., 2006; Lanfranchi \& Duveau, 2008; Vischer, 2007) e cardiovasculares (D. S. Kim \& Kang, 2010; Kraatz et al., 2013; J. Park, Kim, \& Hisanaga, 2011; Van Laethem, Beckers, Kompier, Dijksterhuis, \& Geurts, 2013). Isso torna a alta demanda percebida um dos principais fatores de risco relacionados ao trabalho (Deeney \& O’Sullivan, 2009).

Entretanto, há críticas sobre a excessiva ênfase sobre o modelo Demanda-Controle. A alta demanda e os baixos controle e apoio social não constituem a totalidade dos possíveis riscos psicossociais a serem encontrados nas organizações (Nieuwenhuijsen, Bruinvels, \& Frings-Dresen, 2010). O termo "demandas psicossociais" é inclusive utilizado como sinônimo de "riscos psicossociais", ao se referir que injustiça organizacional e ambiguidade de papéis seriam exemplos de "demandas psicossociais relacionadas ao trabalho" (Kristman et al., 2016). Além disso, o termo job strain tem se tornado construto tão comumente discutido que nem sempre há preocupação em descrevê-lo (Chida \& Steptoe, 2009).

O modelo Effort-Reward Imbalance (ERI), de Siegrist (1996) considera mais os fatores sociais e econômicos do que o modelo Demanda, Controle e Apoio Social (Hoven \& Siegrist, 2013; Kivimäki et al., 2006). Embora fatores de recompensa subjetivos como, por exemplo, o reconhecimento social, são considerados, há grande destaque para níveis de salário, bem como recompensas mais concretas e objetivas (Koch, Schablon, Latza, \& Nienhaus, 2014; Lapointe, Dionne, Brisson, \& Montreuil, 2013; Siegrist, 2011).

O modelo Demanda-Controle e o modelo ERI são não apenas os mais estudados pela literatura (Backé, Seidler, Latza, Rossnagel, \& Schumann, 2012; Hoven \& Siegrist, 2013; Kivimäki et al., 2006), como também são tidos como os modelos com maior poder explicativo a respeito da relação de exposição a riscos psicossociais e doenças. Eles embasam os instrumentos Job Content Questionnaire (JCQ) e Effort-Reward Imbalance Questionnaire (ERI-Q), que são os mais citados e estudados pela literatura científica, inclusive em suas propriedades psicométricas, sendo citados como dotados de alto poder explicativo e preditivo (Bonneterre et al., 2008; Chirico, 2016; Elfering, 2006). Logo, estes dois modelos são bastantes bem-vistos e considerados positivamente pela literatura. Os resultados desta revisão corroboram outras revisões na área, que evidenciam a preferência destes dois modelos quando se abordam riscos psicossociais no trabalho (Fernandes \& Pereira, 2016; Gómez \& Llanos, 2014).

O modelo Demanda-Controle é visto com maior poder explicativo do que o modelo ERI (Hwang \& Hong, 2012), o qual encontra menos poder de associação e predição acerca de doenças relacionadas ao trabalho (Koch et al., 2014). Fatores investigados pelo ERI, como a 
quantidade de esforço empregado e recompensa percebidas, também são associados a agravos à saúde (Bongers et al., 2006; Solovieva, Lallukka, Virtanen, \& Viikari-Juntura, 2013), em especial à saúde mental (Rugulies, Aust, \& Madsen, 2017), e há evidências de que algumas destas associações são de moderadas a baixas (Koch et al., 2014) ou sem significância estatística (Rugulies et al., 2017). Uma das hipóteses é a de que os fatores investigados pelo ERI, isoladamente, têm pouca associação com agravos à saúde; mas combinados, teriam maior potencial para causar agravos à saúde (Bongers et al., 2006). Ainda, o modelo ERI é descrito oras como um outro modelo teórico que explica e influencia processos de job strain (Nieuwenhuijsen et al., 2010), e por vezes como um modelo teórico explicativo de estresse, mas à parte do job strain (Madsen et al., 2017; Schnall et al., 2016). Contudo, tanto o Demanda-Controle quanto o ERI trabalham sob a perspectiva do estresse, com a perspectiva de interação entre dois ou três riscos psicossociais relacionados ao trabalho, e são as teorias proeminentes nestes modelos para a investigação de riscos psicossociais no trabalho (Campos-Serna, RondaPérez, Artazcoz, Moen, \& Benavides, 2013). $\mathrm{Na}$ perspectiva destes modelos, demandas, por si só, não constituem risco, senão o como ela é percebida pelo trabalhador (Eller et al., 2009).

Revisões desde 1980 e até hoje evidenciam que o principal desfecho decorrente da exposição a riscos psicossociais no trabalho é o estresse (Chirico, 2016; Ganster \& Rosen, 2013; International Labour Organization, 1984; Schnall et al., 2016). Em virtude do estresse, agravos à saúde aparecem, sendo os mais estudados as doenças cardiovasculares (Gerin \& James, 2010; Katsarou et al., 2013; D. S. Kim \& Kang, 2010; Kivimäki et al., 2006; J. Park et al., 2011; Pereyra Girardi, Milei, \& Stefani, 2011; Schnall et al., 2016; Thayer et al., 2010) e as musculoesqueléticas (Bovenzi, Schust, \& Mauro, 2017; Deeney \& O’Sullivan, 2009; Jacukowicz, 2016; Koch et al., 2014; Koukoulaki, 2014; Kumar \& Kumar, 2008; Lanfranchi \& Duveau, 2008; Lang, Ochsmann,
Kraus, \& Lang, 2012; J.-K. Park \& Jang, 2010; Violante, Mattioli, \& Bonfiglioli, 2015). Revisões mais recentes trazem também agravos à saúde mental, como burnout (Chirico, 2016) e depressão (Madsen et al., 2017; Rugulies et al., 2017). Outros agravos à saúde com associação a riscos psicossociais no trabalho são doenças hepáticas (Burton, Kendall, Pearce, Birrell, \& Bainbridge, 2009), alterações hormonais (Chida \& Steptoe, 2009), obesidade (Solovieva et al., 2013), suicídio (Nock et al., 2013), fadiga (Turgoose \& Maddox, 2017) e problemas com o sono (Van Laethem et al., 2013).

Apesar da relação consolidada entre estresse e riscos psicossociais (Chirico, 2016; Stravoula Leka et al., 2015; Nieuwenhuijsen et al., 2010), salienta-se que não são termos sinônimos. Há confusão entre agente de risco e agravo proveniente da exposição ao risco (Chirico, 2016). Por vezes, o estresse é referido como um risco psicossocial (Osca et al., 2014; Singh \& Conroy, 2017) ao qual o trabalhador está exposto. O mesmo ocorre com o construto job strain (Hwang \& Hong, 2012; Kraatz et al., 2013; Kristman et al., 2016; Lang et al., 2012; J. Park et al., 2011; Solovieva et al., 2013), que é citado, juntamente com o estresse, como um risco ocupacional, tal como baixo controle e autonomia no trabalho e demandas e exigências elevadas (Hwang \& Hong, 2012; Kristman et al., 2016; Van Laethem et al., 2013). Esta pouca distinção dificulta a tarefa de gerenciamento dos riscos psicossociais no trabalho. Não à toa, os instrumentos de avaliação de riscos psicossociais são criticados por falta de consenso entre o que medem (Bonneterre et al., 2008; Kop et al., 2016; Singh \& Conroy, 2017).

Entende-se que o estresse no trabalho pode ser um fator de risco que contribui para o aparecimento de determinadas doenças (Eller et al., 2009), porém não é um risco ocupacional em si, sendo consequência da exposição a um determinado agente de risco. Esta diferenciação nem sempre é adequada na literatura (Chirico, 2017; Rick et al., 2001), e é mais comum quando se investigam riscos psicossociais sob os termos "estressores 
psicossociais" (Lang et al., 2012; Van Laethem et al., 2013) ou "fatores psicossociais" (Kraatz et al., 2013; J. Park et al., 2011; Solovieva et al., 2013). Salientam-se, aí, considerações a respeito do uso destes dois termos.

Conceitualmente, fatores psicossociais podem ser positivos ou negativos, apesar de serem geralmente associados a elementos que prejudicam a saúde (ILO, 1984). Uma minoria de revisões desta amostra selecionada trabalha com o conceito de fatores de proteção, ou protetivos, ou promotores de saúde (Boccio \& Macari, 2014; S. A. Kim, Oh, Suh, \& Seo, 2014; Kristman et al., 2016; Nock et al., 2013). O termo "fatores psicossociais" continua sendo utilizado principalmente como sinônimo de fatores de risco, corroborando a acepção da ILO de 1984 (Backé et al., 2012; Burton et al., 2009; Gray et al., 2011; D. S. Kim \& Kang, 2010; Koch et al., 2014; J.-K. Park \& Jang, 2010; Silva, Barros, Cunha, Carnide, \& Santos, 2014; Solovieva et al., 2013).

Quando se utiliza o termo "fator" ou "fator de risco psicossocial", nem sempre há clareza sobre as variáveis que estão em investigação. Podem se tratar de aspectos individuais, do ambiente, do resultado da interação entre estes dois aspectos, de consequências da exposição a estes riscos, de elementos que contribuem para determinado agravo à saúde (Singh \& Conroy, 2017) ou a todas estas questões simultaneamente (Hwang \& Hong, 2012; Nock et al., 2013; Thayer et al., 2010). Logo, nem todo "fator psicossocial" se refere a elementos ocupacionais (Farnacio et al., 2017).

Características individuais referidas pelo termo "fator psicossocial" envolvem tanto componentes psicológicos, como coping, traços de personalidade e cognição; até características sociodemográficas como sexo, idade, religião, status e condição familiar e social, perpassando também por histórico clínico-médico de doenças hereditárias e condições de saúde física e mental (Lanfranchi \& Duveau, 2008; Nock et al., 2013; Siegrist, 2011; Turgoose \& Maddox, 2017). Ainda, ao se discutir "fatores psicossociais", pode se falar de: riscos psicossociais ocupacionais; riscos psicossociais relacionados ao trabalho, porém não ocupacionais (Hoven \& Siegrist, 2013; Kivimäki et al., 2006; Siegrist, 2011); e riscos psicossociais de ambientes fora do trabalho (Villalobos et al., 2013). Com fatores psicossociais, também se encontra referência ao fenômeno do estresse em si, e até mesmo de agravos à saúde mental que podem advir do estresse, como depressão e ansiedade (Katsarou et al., 2013). Este termo tem sido utilizado para descrever todos os elementos que não são físicos e diretamente observáveis associados ao ambiente de trabalho (Deeney \& O’Sullivan, 2009).

Outra consideração é a respeito do termo "estressor psicossocial". Processos de estresse se referem a qualquer elemento percebido que o indivíduo considere estressor. Estressores, na vida de um indivíduo, podem ser ocupacionais ou relacionados ao trabalho, porém não ocupacionais, como, por exemplo, mercado e condições de trabalho (Kivimäki et al., 2006; Schnall et al., 2016), relação trabalho-família (Rugulies et al., 2017), condições socioeconômicas de contexto no qual as organizações do trabalho estão inseridas (Hoven \& Siegrist, 2013; Siegrist, 2011) e até mesmo do trânsito (Eller et al., 2009). Estressores podem ser provenientes também de contextos sem relação estreita com o trabalho: financeiros, familiares, matrimoniais, ou do cotidiano da vida (Farnacio et al., 2017; Katsarou et al., 2013; Lanfranchi \& Duveau, 2008; Schnall et al., 2016). Podem ser de situações atuais ou passadas, como, por exemplo, experiências de vida e traumas (Nock et al., 2013). Ainda, há os estressores que são de ordem social, da época vigente, comuns à sociedade moderna, sobre os quais as pessoas têm pouco controle direto; como o avanço da globalização e da tecnologia, excesso de estímulos cognitivos e processos de tomada de decisão cada vez mais intrincados (Belkić \& Nedić, 2014; Hoven \& Siegrist, 2013). O organismo reage ao estresse da mesma forma, independentemente da origem do estressor - se é ocupacional, doméstico, 
familiar, ou de condições sociais e econômicas (Campos-Serna et al., 2013; Ilies et al., 2016; Vischer, 2007).

No contexto ocupacional, estressores podem ser de ordem física, química, biológica, de acidentes, ou mesmo psicossociais (Rashid \& Zimring, 2008). Ainda que um estressor seja ocupacional, ele não necessariamente é psicossocial, visto que qualquer agente de risco que perturbe a homeostase do indivíduo é um estressor. Ainda que se queira enfatizar perspectivas cognitivas, com o intuito de afirmar que a "natureza do estressor é psicossocial" porque o processo pelo qual ele é percebido é de natureza psicossocial (isto é, é um elemento percebido do ambiente processado pela cognição do indivíduo), isto é uma característica do processo do estresse e não do agente de risco em si. Este tipo de diálogo confunde e dificulta a tarefa de investigação de riscos psicossociais no trabalho de forma mais objetiva, inclusive em se tratando de perceber quais variáveis constituem o construto riscos psicossociais no trabalho (Weissbrodt \& Giauque, 2017).

A diferença fundamental entre modelos clássicos, pautados na teoria do job strain e do estresse, e modelos emergentes, situase principalmente na diversidade de riscos psicossociais identificados no contexto ocupacional que estes apresentam em relação a aqueles. O escopo aberto por Siegrist, no intuito de sinalizar que há outras variáveis de risco além daquelas previstas pelo modelo job strain (Hoven \& Siegrist, 2013; Siegrist, 2011), é avançado por outros estudos organizacionais referentes à importância de aspectos como contrato de trabalho, qualidade de liderança, relações interpessoais (Kivimäki et al., 2006; Peiró \& Rodríguez, 2008), clima de segurança, bullying, relação trabalho e família, perspectiva de carreira, competitividade, papéis laborais, insegurança no trabalho, remuneração, conteúdo e variedade de tarefas, e aspectos éticos nas relações de trabalho (Eller et al., 2009; Kristman et al., 2016; Nieuwenhuijsen et al., 2010; Schnall et al., 2016).
Destacam-se o modelo de Justiça Organizacional e o modelo psicossocial de Copenhagen como expoentes.

O modelo de Justiça Organizacional se baseia no pressuposto de que o clima organizacional da empresa é diretamente influenciado pelo tratamento interpessoal justo, pela confiança e reciprocidade nas relações humanas; bem como pela aplicação zelosa e imparcial das normas organizacionais (Lang et al., 2012; Peiró \& Rodríguez, 2008; Van Laethem et al., 2013). Estudos referentes à justiça organizacional são encontrados antes dos anos 2000, mas foi a partir dos estudos de Whitehall II, em 2005, que houve a associação entre aspectos deste modelo teórico e o estresse organizacional, tornando-a um dos modelos teóricos emergentes de riscos psicossociais no trabalho (Kivimäki et al., 2006; Rugulies et al., 2017). É referido como um modelo emergente porque há poucos estudos comprovando seu caráter preditor no adoecimento do trabalhador, inclusive em função de ser um modelo mais recente, quando comparado com os demais (Bonneterre et al., 2008; Eller et al., 2009; Ganster \& Rosen, 2013).

O modelo psicossocial de Copenhagen é referido como sendo um dos modelos mais completos na investigação de aspectos psicossociais, e um dos mais adequados para embasar estratégias de saúde e segurança ocupacional, pela diversidade de riscos que investiga (Chirico, 2016; Deeney \& O'Sullivan, 2009; Villalobos et al., 2013). Este modelo embasa um dos instrumentos mais referidos na literatura, após o JCQ e o ERI, que é o Copenhagen Psychosocial Questionnaire (COPSOQ) (Koch et al., 2014). Já há uma segunda versão do questionário (Madsen et al., 2017), que inclusive engloba dimensões como respeito, justiça e conflitos emocionais como fatores psicossociais a serem avaliados (Burr, Pedersen, \& Hansen, 2011; Chirico, 2016).

Uma das diferenças significativas nos modelos de gestão é referir-se aos riscos psicossociais como riscos ocupacionais a 
serem gerenciados e minimizados, capazes de provocar acidentes e doenças tanto quanto riscos ocupacionais mais visíveis e consolidados na literatura em saúde e segurança do trabalho (Moraru, Băbuţ, \& Popescu Stelea, 2014; Zoni \& Lucchini, 2012). Desta forma, o termo "risco psicossocial" (psychosocial risk e hazard, em inglês) é utilizado com mais frequência do que termos como psychosocial stressor, factor ou mesmo risk factor.

Se nos modelos emergentes, outros riscos psicossociais além da demanda e do controle são considerados, em modelos de gestão há mais avanços e, inclusive, preocupação com embasamentos legais. Aspectos do cotiano do trabalho já são considerados, como registro de horário de trabalho, licenças saúde e maternidade, combate às diferentes formas de discriminação e preconceito no trabalho. Salienta-se a necessidade de condutas humanizadoras, justas e respeitosas no ambiente de trabalho,n além de inclusão dos trabalhadores nas tomadas de decisão, progressão de carreira, respeito às minorias e às diferenças de gênero (Burr et al., 2011; Zoni \& Lucchini, 2012). A crítica ao que era abstrato e subjetivo nos modelos anteriores ganha caráter mais prático e aplicado nesses modelos (Biron \& KaranikaMurray, 2014).

Modelos de gestão buscam associar não apenas a minimização de riscos psicossociais no trabalho com agravos à saúde, mas também com o impacto organizacional negativo das perdas de produtividade e elevação de custos (Burr et al., 2011; Koukoulaki, 2014). Há preocupações, também, com que os riscos psicossociais sejam identificados exclusivamente por meio da percepção dos trabalhadores (Hwang \& Hong, 2012; Koukoulaki, 2014; Kristman et al., 2016; Singh \& Conroy, 2017) e, para tanto, os modelos de gestão enfatizam medidas e parâmetros mais objetivos (Zoni \& Lucchini, 2012).

A ênfase no aspecto cognitivo - presente nas teorias do estresse, em especial no modelo transacional - é minimizada, em parte pela questão do viés do trabalhador e, por outro lado, porque gerir riscos ocupacionais significa monitorar indicadores objetivos do ambiente de trabalho (Biron \& Karanika-Murray, 2014; Koukoulaki, 2014). Tal perspectiva visa a corroborar o conceito de "risco psicossocial" como risco ocupacional, com potencial intrínseco para causar dano, independentemente da interação com aspectos individuais (International Labour Organization, 2017; Kumar \& Kumar, 2008). Frente a isso, tenta-se reduzir o viés do risco percebido pelo uso do termo "risco psicossocial relacionado ao trabalho", ao invés de "fator psicossocial". Os termos hazard e risks são utilizados, ao invés de stressors (estressores), quando se aborda tais agentes (Rick et al., 2001). Essa perspectiva representa um corte importante com teorias do estresse clássicas, incluindo as perspectivas modernas de Karasek e Siegrist, por caracterizar riscos psicossociais como agentes que existem no ambiente independentemente da percepção.

Não surpreende a aproximação da Ergonomia com a investigação de riscos psicossociais, sob a ótica de gestão de saúde ocupacional (Boccio \& Macari, 2014; Lang et al., 2012; Zare, Croq, Hossein-Arabi, Brunet, \& Roquelaure, 2016). O foco de estudo da Ergonomia tende a ser os riscos mais diretamente observáveis, contra os quais os riscos psicossociais encontram relativa invisibilidade e menor poder de explicação de doenças (Kim \& Kang, 2010; Moraru et al., 2014; Waters, Genaidy, Viruet, \& Makola, 2008; Zare et al., 2016). Contudo, há evidências de que são tão ou mais responsáveis pelo adoecimento musculoesquelético dos trabalhadores do que a sobrecarga física de trabalho, o que fortalece a necessidade de se gerir esse tipo de risco (Gray et al., 2011; Lanfranchi \& Duveau, 2008; J.-K. Park \& Jang, 2010; J. Park et al., 2011; Silva et al., 2014).

Um dos termos utilizados para tentar definir estes riscos "invisíveis" é o termo "ambiente de trabalho psicossocial" (psychosocial work environment). Um ambiente de trabalho psicossocialmente pobre é definido por condições muitas vezes intangíveis e nem sempre fáceis de 
verificação, como: relações interpessoais, injustiça social, comportamentos negativos e/ou agressivos, remoção de responsabilidades e competências, exclusão social (Burr et al., 2011; Skogstad et al., 2011; Weissbrodt \& Giauque, 2017). Pode-se encontrar, ainda, o termo "condições psicossociais do ambiente de trabalho" e outros similares.

O termo risco psicossocial foi trazido à tona por estudiosos de programas de gerenciamento de riscos psicossociais, destacando-se o PRIMAEF (Stravoula Leka, Jain, Cox, \& Kortum, 2011; Stravoula Leka et al., 2015). Trata-se, provavelmente, do programa de gerenciamento de riscos psicossociais no trabalho mais notório. Outros programas também se destacaram: o Management Standard for Work-Related Stress (Inglaterra), START (Alemanha); SOBANE (Screening, Observation, Analysis, Expertise Bélgica) e INAIL-ISPESL (National Institute for Prevention and Safety at Work - Itália) (Bonneterre et al., 2008; Chirico, 2016; Stravoula Leka et al., 2015; Villalobos et al., 2013). Os modelos de gerenciamento mais referidos são europeus, muito em função do avanços no desenvolvimento de políticas e legislações sobre riscos psicossociais no trabalho (Deeney \& O’Sullivan, 2009; Leka et al., 2011, 2015; Zoni \& Lucchini, 2012). Há a necessidade de revisão de aspectos destas legislações, pois gerenciar riscos psicossociais deve resultar em melhorias concretas e não ser apenas uma discussão de ideias (Biron \& Karanika-Murray, 2014; Peiró \& Rodríguez, 2008; Weissbrodt \& Giauque, 2017). Tanto o termo "fator de risco psicossocial", quanto o termo "risco psicossocial" são utilizados pelas principais agências e referências internacionais (International Labour Organization, 2017; Leka, \& Cox, 2008; Leka, Jain, Iavicoli, \& Di Tecco, 2015). Salienta-se a necessidade de saber utilizálos, ciente de seu significado, sem tomá-los como sinônimos. Entende por riscos (hazards/ risks) psicossociais as características da gestão e da organização do trabalho com potencial nocivo à saúde do trabalhador (Farnacio et al., 2017; Stravoula Leka et al., 2011). Ainda que a resposta de cada trabalhador varie, o potencial nocivo é intrínseco ao trabalho. Fator psicossocial (psychosocial factor) compreende que o risco ao qual trabalhador está exposto terá um efeito modificado pela influência de suas características pessoais ou subjetivas (Park \& Jang, 2010; Zoni \& Lucchini, 2012). Logo, o fator não é o risco em si, mas sim o risco processado, com potencial para ser protetivo (protective factor) ou nocivo (risk factor) (Nock et al., 2013).

As características pessoais ou subjetivas podem ser de natureza biológica ou psicológica (Bekker, Rutte, \& Van Rijswijk, 2009; Turgoose \& Maddox, 2017), que envolve um condição geral de saúde e ou de interação no grupo de trabalho (Skogstad et al., 2011). Elas influenciam o modo como o trabalhador percebe e avalia o risco, assim como o predispõe ao estresse (Eddy, Wertheim, Kingsley, \& Wright, 2017; Pereyra Girardi et al., 2011; Rashid \& Zimring, 2008; Szerencsi et al., 2012). A avaliação de riscos requer que seja descrito o modo como o risco é avaliado, pois há distinção entre resultados daquilo que é mensurado objetivamente no ambiente e o que é avaliado por meio da percepção do trabalhador, ainda que os dois métodos possam ser combinados (Bonneterre et al., 2008; Burton et al., 2009).

É importante avaliar tanto os riscos psicossociais, que são as características de gestão e processo de trabalho que agridem a saúde (Post Sennehed et al., 2017; Shaw, van der Windt, Main, Loisel, \& Linton, 2009), quanto os fatores de risco psicossociais, que são o produto da resposta do trabalhador frente aos riscos, mediante suas capacidades individuais de enfrentamento (Park \& Jang, 2010; Rashid \& Zimring, 2008) são conceitos que necessitam de aprimoramento conceitual e de instrumentação dimensionada à suas distinções e escopo de validade.

No plano prático, contudo, é cada vez saliente a necessidade de se avaliar tanto os riscos psicossociais quanto os riscos ocupacionais, tendo em vista a finalidade e especificidades em seu emprego (Moraru et al., 2014). Isso envolve a avaliação de riscos e de fatores de 
riscos relacionados de natureza ocupacional ou provenientes de outros contextos, distante do escopo de gerenciamento da organização (Belkić \& Nedić, 2014; Bovenzi et al., 2017; Peiró \& Rodríguez, 2008). Entretanto, nem sempre o risco psicossocial ocupacional pode ser avaliado objetivamente por meio de instrumentos objetivos, sendo acessível, às vezes, somente pelo relato do trabalhador (Skogstad et al., 2011). Entretanto, é necessário o cuidado na magnitude da influência de aspectos pessoais ou subjetivos na avaliação de riscos, a fim de minimizar variáveis de confusão (Belkić \& Nedić, 2014).

Assim, com base no estudo da literatura especializada, é possível definir riscos psicossociais ocupacionais como exigências ou condições de trabalho com potencial para provocar constrangimentos e danos pessoais (Stravoula Leka et al., 2015; Weissbrodt \& Giauque, 2017). São originários das condições de trabalho, têm, portanto, delimitação no ambiente ocupacional e implicam na capacidade da organização em gerenciá-los (Moraru et al., 2014; Biron \& Karanika-Murray, 2014; Weissbrodt \& Giauque, 2017). Utilizar este termo é diferente de se trabalhar com riscos psicossociais relacionados ao trabalho, ainda que este último seja o mais referido na literatura. Riscos psicossociais relacionados ao trabalho não necessariamente emergem do ambiente ocupacional, mas de outros ambientes sociais pelos quais o trabalhador transita, e o processo de gerenciamento de riscos provenientes destas fontes é mais delicado e intangível para a organização. Já os riscos psicossociais ocupacionais são parte da agenda de gestão de riscos ocupacionais da organização.

\section{Conclusões}

O conceito mais utilizado na discussão de riscos psicossociais ainda é o "fator psicossocial", que se relaciona com o conceito clássico trazido pela ILO, o qual enfatiza que tais riscos são um produto da interação entre características do ambiente e características individuais do trabalhador. Ressalta-se, contudo, que, cada vez mais, busca-se explicar riscos psicossociais por de critérios mais objetivos, entendendo e avaliando riscos psicossociais como riscos ocupacionais (Moraru et al., 2014; Waters et al., 2008; Zare et al., 2016). O termo estressores psicossociais ainda é bastante utilizado (Ganster \& Rosen, 2013), apesar de críticas de que este termo desconsidera uma gama de estressores do ambiente de trabalho que não tem natureza psicossocial (Backé et al., 2012; Kraatz et al., 2013). Nas perspectivas do estresse, agir deste modo é reduzir o escopo do estudo de estressores ocupacionais, que podem ser de diversas ordens: físicos, químicos, biológicos (Park et al., 2011).

$\mathrm{Na}$ perspectiva de gestão de riscos ocupacionais, estudar riscos psicossociais com origem ocupacional e diferenciá-los de outros riscos ocupacionais, e de riscos psicossociais não relacionados ao trabalho, é uma inovação e um desafio (Stravoula Leka et al., 2011, 2015). Estresse, violência e bullying podem ser considerados resultantes da exposição a estes riscos (Koukoulaki, 2014); logo, trabalhar para minimizar essas consequências tem pouco efeito, senão trabalhar para erradicar os agentes de risco ocupacional. Perspectivas de gestão enfatizam que determinadas características do ambiente possuem potencial próprio para causar danos à saúde, como qualquer outro risco ocupacional (Lapointe et al., 2013; Taylor, Goode, George, \& Cook, 2014; Violante et al., 2015), independentemente da influência da subjetividade do trabalhador (Burton et al., 2009; Hasle, 2014; Waters et al., 2008).

Apesar de que as teorias do estresse são as mais proeminentes no estudo de riscos psicossociais, é importante considerar a ênfase dessas teorias nas características percebidas do que em riscos reais no ambiente de trabalho (Belkić \& Nedić, 2014; Eddy et al., 2017). Modelos teóricos mais recentes têm trabalhado com a investigação de aspectos objetivos e mensuráveis no ambiente de trabalho. Estudar riscos psicossociais encontra forte relação com as práticas em segurança do 
trabalho, que visa a minimizar riscos ocupacionais aos quais os trabalhadores estão expostos (Kumar \& Kumar, 2008; Stravoula Leka et al., 2015; Weissbrodt \& Giauque, 2017; Zare et al., 2016); ao passo em que estudar fatores de risco psicossociais no trabalho ainda é relevante sob a ótica da saúde do trabalhador, que adoece e sofre mediante exposição a determinados riscos (Kristman et al., 2016; Nieuwenhuijsen et al., 2010; Rugulies et al., 2017; Singh \& Conroy, 2017).

Esta revisão procurou avançar na análise crítica dos diferentes termos para nomear riscos psicossociais no trabalho e suas bases teóricas. Contudo, fatores, fatores de risco, riscos, condições psicossociais de trabalho e estressores ainda são tratados como termos sinônimos (Eller et al., 2009; Villalobos et al., 2013). Sugere-se aprofundamento dos estudos em termos metodológicos, procurando discutir a operacionalização de construtos e de métodos de mensuração de riscos psicossociais e sua consistência em produzir conhecimento relevante ao estudo dos fatores humanos no trabalho. Também se sugere a melhor investigação de fatores de proteção psicossocial no trabalho, conceito discutido há décadas, mas ainda embrionário em termos de discussão na literatura (Boccio \& Macari, 2014; Ilies et al., 2016; Nock et al., 2013), em comparação com fatores de risco psicossociais no trabalho.

\section{Referências}

Aghaei Chadegani, A., Salehi, H., Md Yunus, M. M., Farhadi, H., Fooladi, M., Farhadi, M., \& Ale Ebrahim, N. (2013). A comparison between two main academic literature collections: Web of science and scopus databases. Asian Social Science, 9(5), 1826. https://doi.org/10.5539/ass.v9n5p18

Backé, E.-M., Seidler, A., Latza, U., Rossnagel, K., \& Schumann, B. (2012). The role of psychosocial stress at work for the development of cardiovascular diseases: A systematic review. International Archives of Occupational and Environmental Health, 85(1). https://doi.org/10.1007/s00420-0110643-6

Bekker, M. H. J., Rutte, C. G., \& Van Rijswijk, K. (2009). Sickness absence: A genderfocused review. Psychology Health \& Medicine, 14(4), 405-418. https://doi. org/10.1080/13548500903012830

Belkić, K., \& Nedić, O. (2014). Occupational medicine-then and now: where we could go from here. Medicinski Pregled, 67(56), 139-147. Retrieved from https://www. scopus.com/inward/record.uri?eid=2-s2.0$85005865090 \&$ partnerID $=40 \&$ md $5=6 \mathrm{cde} 9 \mathrm{~b}$ $40 f 4 d 3 d b 8 f 26547 \mathrm{c} 79 f 88923 \mathrm{bd}$

Biron, C., \& Karanika-Murray, M. (2014). Process evaluation for organizational stress and wellbeing interventions: Implications for theory, method, and practice. International Journal of Stress Management, 21(1), 85-111. https:// doi.org/10.1037/a0033227

Boccio, D. E., \& Macari, A. M. (2014). Workplace as Safe Haven: How Managers Can Mitigate Risk for Employee Suicide. Journal of Workplace Behavioral Health, 29(1). https:// doi.org/10.1080/15555240.2014.866472

Bongers, P. M., Ijmker, S., Van Den Heuvel, S., \& Blatter, B. M. (2006). Epidemiology of work related neck and upper limb problems: Psychosocial and personal risk factors (Part I) and effective interventions from a bio behavioural perspective (Part II). Journal of Occupational Rehabilitation, 16(3), 279302. https://doi.org/10.1007/s10926-0069044-1

Bonneterre, V., Liaudy, S., Chatellier, G., Lang, T., \& De Gaudemaris, R. (2008). Reliability, validity, and health issues arising from 
questionnaires used to measure psychosocial and organizational work factors (POWFs) among hospital nurses: A critical review. Journal of Nursing Measurement, 16(3). https://doi.org/10.1891/1061-3749.16.3.207

Bovenzi, M., Schust, M., \& Mauro, M. (2017). An overview of low back pain and occupational exposures to whole-body vibration and mechanical shocks. Medicina Del Lavoro, 108(6), 419-433. https://doi.org/10.23749/ mdl.v108i6.6639

Burr, H., Pedersen, J., \& Hansen, J. V. (2011). Work environment as predictor of long-term sickness absence: Linkage of self-reported DWECS data with the DREAM register. Scandinavian Journal of Public Health, 39(7). https://doi.org/10.1177/1403494811401480

Burton, A. K., Kendall, N. A. S., Pearce, B. G., Birrell, L. N., \& Bainbridge, L. C. (2009). Management of work-relevant upper limb disorders: A review. Occupational Medicine, 59(1), 44-52. https://doi.org/10.1093/ occmed/kqn 151

Campos-Serna, J., Ronda-Pérez, E., Artazcoz, L., Moen, B. E., \& Benavides, F. G. (2013). Gender inequalities in occupational health related to the unequal distribution of working and employment conditions: A systematic review. International Journal for Equity in Health, 12(1). https://doi.org/10.1186/14759276-12-57

Chida, Y., \& Steptoe, A. (2009). Cortisol awakening response and psychosocial factors: A systematic review and metaanalysis. Biological Psychology, 80(3), 265-278. https://doi.org/10.1016/j. biopsycho.2008.10.004

Chirico, F. (2016). Job stress models for predicting burnout syndrome: a review. Ann Ist Super Sanità, 52(3), 443-456. https://doi. org/10.4415/ANN_16_03_17
Chirico, F. (2017). The forgotten realm of the new and emerging psychosocial risk factors. Journal of Occupational Health, 59(5), 433435. https://doi.org/10.1539/joh.17-0111-OP

Deeney, C., \& O'Sullivan, L. (2009). Work related psychosocial risks and musculoskeletal disorders: Potential risk factors, causation and evaluation methods. Work, 34(2), 239248. https://doi.org/10.3233/WOR-20090921

Eddy, P., Wertheim, E. H., Kingsley, M., \& Wright, B. J. (2017). Associations between the effort-reward imbalance model of workplace stress and indices of cardiovascular health: A systematic review and metaanalysis. Neuroscience and Biobehavioral Reviews, 83(October), 252-266. https://doi. org/10.1016/j.neubiorev.2017.10.025

Elfering, A. (2006). Work-related outcome assessment instruments. European Spine Journal, 15(1), S32-S43. https://doi. org/10.1007/s00586-005-1047-7

Eller, N. H., Netterstrøm, B., Gyntelberg, F., Kristensen, T. S., Nielsen, F., Steptoe, A., \& Theorell, T. (2009). Work-related psychosocial factors and the development of ischemic heart disease: A systematic review. Cardiology in Review, 17(2), 83-97. https:// doi.org/10.1097/CRD.0b013e318198c8e9

Farnacio, Y., Pratt, M. E., Marshall, E. G., \& Graber, J. M. (2017). Are Workplace Psychosocial Factors Associated with Work-Related Injury in the US Workforce?: National Health Interview Survey, 2010. Journal of Occupational and Environmental Medicine, 59(10), e164-e171. https://doi. org/10.1097/JOM.0000000000001143

Fernandes, C., \& Pereira, A. (2016). Exposure to psychosocial risk factors in the context of work: a systematic review. Revista de Saude Publica, 50. https://doi.org/10.1590/S15188787.2016050006129 
Ganster, D. C., \& Rosen, C. C. (2013). Work Stress and Employee Health: A Multidisciplinary Review. Journal of Management, 39(5), 1085-1122. https://doi. org/10.1177/0149206313475815

Gerin, W., \& James, G. D. (2010). Psychosocial determinants of hypertension: Laboratory and field models. Blood Pressure Monitoring, 15(2), 93-99. https://doi.org/10.1097/ MBP.0b013e3283380ea

Gómez, G. F. V., \& Llanos, R. A. (2014). Factores psicosociales de origen laboral, estrés y morbilidad en el mundo. Psicología Desde El Caribe, 31(2), 354-385. https://doi. org/2011-7485

Gray, H., Adefolarin, A. T., \& Howe, T. E. (2011). A systematic review of instruments for the assessment of work-related psychosocial factors (Blue Flags) in individuals with nonspecific low back pain. Manual Therapy, 16(6), 531-543. https://doi.org/10.1016/j. math.2011.04.001

Hasle, P. (2014). Lean Production-An Evaluation of the Possibilities for an Employee Supportive Lean Practice. Human Factors and Ergonomics In Manufacturing \& Service Industries, 24(1), 40-53. https://doi. org/10.1002/hfm.20350

Hoven, H., \& Siegrist, J. (2013). Work characteristics, socioeconomic position and health: a systematic review of mediation and moderation effects in prospective studies. Occupational and Environmental Medicine, 70(9), 663-669. https://doi.org/10.1136/ oemed-2012-101331

Hwang, W. J., \& Hong, O. (2012). Work-related cardiovascular disease risk factors using a socioecological approach: Implications for practice and research. European Journal of Cardiovascular Nursing, 11(1). https://doi. org/10.1177/1474515111430890
Ilies, R., Aw, S. S. Y., \& Lim, V. K. G. (2016). A Naturalistic Multilevel Framework for Studying Transient and Chronic Effects of Psychosocial Work Stressors on Employee Health and Well-Being. Applied Psychology, 65(2), 223-258. https://doi.org/10.1111/ apps. 12069

International Labour Organization. (1984). Psychosocial Factors At Work : Recognition and Control. Safety And Health (Vol. 13). https://doi.org/10.1016/S10903801(09)60542-5

International Labour Organization. (2017). Workrelated Stress and Psychosocial Risks. (P. and D. B. (PRODOC) of the I. Document and Publications Production, Ed.) (8th ed.). Geneva: International Labour Office. Retrieved from http://www.ilo.org/actrav/ info/international-journal-labour-research/ WCMS_551796/lang--en/index.htm

Jacukowicz, A. (2016). Psychosocial work aspects, stress and musculoskeletal pain among musicians. A systematic review in search of correlates and predictors of playingrelated pain. Work: Journal of Prevention, Assessment \& Rehabilitation, 54(3), 657668. https://doi.org/10.3233/WOR-162323

Katsarou, A. L., Triposkiadis, F., \& Panagiotakos, D. (2013). Perceived stress and vascular disease: Where are we now? Angiology, 64(7), 529-534. https://doi. org/10.1177/0003319712458963

Kim, D. S., \& Kang, S. K. (2010). Work-related cerebro-cardiovascular diseases in Korea. Journal of Korean Medical Science, 25(SUPPL.1). https://doi.org/10.3346/ jkms.2010.25.S.S105

Kim, S. A., Oh, H. S., Suh, Y. O., \& Seo, W. S. (2014). An integrative model of workplace self-protective behavior for Korean nurses. Asian Nursing Research, 8(2), 91-98. https:// doi.org/10.1016/j.anr.2014.05.003 
Kivimäki, M., Virtanen, M., Elovainio, M., Kouvonen, A., Väänänen, A., \& Vahtera, J. (2006). Work stress in the etiology of coronary heart disease - A meta-analysis. Scandinavian Journal of Work, Environment and Health, 32(6).

Koch, P., Schablon, A., Latza, U., \& Nienhaus, A. (2014). Musculoskeletal pain and effortreward imbalance - A systematic review. BMC Public Health, 14(1). https://doi. org/10.1186/1471-2458-14-37

Kop, J.-L., Althaus, V., Formet-Robert, N., \& Grosjean, V. (2016). Systematic comparative content analysis of 17 psychosocial work environment questionnaires using a new taxonomy. International Journal of Occupational \& Environmental Health, 22(2), 128-141. https://doi.org/10.1080/107 73525.2016 .1185214

Koukoulaki, T. (2014). The impact of lean production on musculoskeletal and psychosocial risks: An examination of sociotechnical trends over 20 years. Applied Ergonomics, 45(2 Part A). https://doi. org/10.1016/j.apergo.2013.07.018

Kraatz, S., Lang, J., Kraus, T., Münster, E., \& Ochsmann, E. (2013). The incremental effect of psychosocial workplace factors on the development of neck and shoulder disorders: A systematic review of longitudinal studies. International Archives of Occupational and Environmental Health, 86(4). https://doi. org/10.1007/s00420-013-0848-y

Kristman, V. L., Shaw, W. S., Boot, C. R. L., Delclos, G. L., Sullivan, M. J., Ehrhart, M. G., \& Grp, H. C. W. (2016). Researching Complex and Multi-Level Workplace Factors Affecting Disability and Prolonged Sickness Absence. Journal of Occupational Rehabilitation, 26(4, SI), 399-416. https:// doi.org/10.1007/s10926-016-9660-3
Kumar, R., \& Kumar, S. (2008). Musculoskeletal risk factors in cleaning occupation - A literature review. International Journal of Industrial Ergonomics, 38(2), 158-170. https://doi.org/10.1016/j.ergon.2006.04.004

Laisné, F., Lecomte, C., \& Corbière, M. (2012). Biopsychosocial predictors of prognosis in musculoskeletal disorders: A systematic review of the literature (corrected and republished). Disability and Rehabilitation, 34(22), 1912-1941. https://doi.org/10.3109/ 09638288.2012 .729362

Lanfranchi, J.-B., \& Duveau, A. (2008). Explicative models of musculoskeletal disorders (MSD): From biomechanical and psychosocial factors to clinical analysis of ergonomics. Revue Europeenne de Psychologie Appliquee, 58(4), 201-213. https://doi.org/10.1016/j.erap.2008.09.004

Lang, J., Ochsmann, E., Kraus, T., \& Lang, J. W. B. (2012). Psychosocial work stressors as antecedents of musculoskeletal problems: A systematic review and meta-analysis of stability-adjusted longitudinal studies. Social Science and Medicine, 75(7). https:// doi.org/10.1016/j.socscimed.2012.04.015

Lapointe, J., Dionne, C. E., Brisson, C., \& Montreuil, S. (2013). Effort-reward imbalance and video display unit postural risk factors interact in women on the incidence of musculoskeletal symptoms. Work, 44(2). https://doi.org/10.3233/WOR-2012-1357

Leka,S.,\&Cox,T.(2008).TheEuropeanframework for psychosocial risk management: PRIMAEF. (S. Leka \& T. Cox, Eds.), PRIMA-EF. I-WHO Publications, Nottingham. WHO Publications (C) https://doi.org/10.1539/joh. O10010

Leka, S., Jain, A., Cox, T., \& Kortum, E. (2011). The development of the European framework for psychosocial risk management: PRIMA- 
EF. Journal of Occupational Health, 53(2), 137-143. https://doi.org/10.1539/joh. O10010

Leka, S., Jain, A., Iavicoli, S., \& Di Tecco, C. (2015). An Evaluation of the Policy Context on Psychosocial Risks and Mental Health in the Workplace in the European Union: Achievements, Challenges, and the Future. BioMed Research International, 2015. https://doi.org/10.1155/2015/213089

Madsen, I. E. H., Nyberg, S. T., Magnusson Hanson, L. L., Ferrie, J. E., Ahola, K., Alfredsson, L., ... Kivimäki, M. (2017). Job strain as a risk factor for clinical depression: systematic review and meta-analysis with additional individual participant data. Psychological Medicine, 47(08), 1342-1356. https://doi. org/10.1017/S003329171600355X

Moraru, R. I., Băbuţ, G. B., \& Popescu Stelea, M. (2014). Prerequisites for structuring and developing a Romanian occupational stress assessment tool. Quality - Access to Success, 15(143).

Nieuwenhuijsen, K., Bruinvels, D., \& FringsDresen, M. (2010). Psychosocial work environment and stress-related disorders, a systematic review. Occupational Medicine (Oxford, England), 60(4), 277-286. Retrieved from https://www.scopus.com/inward/ record.uri?eid=2-s2.0-79951945612\&partne $\mathrm{rID}=40 \& \mathrm{md} 5=44 \mathrm{f} 3 \mathrm{~b} 19031 \mathrm{~d} 33074 \mathrm{~cd} 4 \mathrm{bf6f3}$ 36b0d487

Nock, M. K., Deming, C. A., Fullerton, C. S., Gilman, S. E., Goldenberg, M., Kessler, R. C., ... Ursano, R. J. (2013). Suicide among soldiers: A review of psychosocial risk and protective factors. Psychiatry (New York), 76(2), 97-125. https://doi.org/10.1521/ psyc.2013.76.2.97

Organización Internacional del Trabajo. (2010). Riesgos emergentes y nuevos modelos de prevención en un mundo de trabajo en transformación, 22. https://doi.org/978-922-323343-3

Organización Internacional del Trabajo. (2013). La organización del trabajo y los riesgos psicosociales: una mirada de género, 3,8 . Retrieved from www.oit.or.cr

Osca, A., López-Araujo, B., Bardera, P., Uríen, B., Díez, V., \& Rubio, C. (2014). Psychosocial risks and accidents: Research and proposals for action. Papeles Del Psicologo, 35(2), 138-143.

Oxford University Press. (2018). English Oxford Living Dictionaries. Retrieved November 1, 2017, from https://en.oxforddictionaries. com/

Park, J.-K., \& Jang, S.-H. (2010). Association between upper extremity musculoskeletal disorders and psychosocial factors at work: A review on the job DCS model's perspective. Safety and Health at Work, 1(1), 37-42. https://doi.org/10.5491/SHAW.2010.1.1.37

Park, J., Kim, Y., \& Hisanaga, N. (2011). Workrelated cerebrovascular and cardiovascular diseases (WR-CVDs) in Korea. Industrial Health, 49(1), 3-7. https://doi.org/10.2486/ indhealth.MS1191

Peiró, J. M., \& Rodríguez, I. (2008). Work stress, leadership and organizational health. Papeles Del Psicologo, 29(1), 68-82. Retrieved from https://www.scopus.com/inward/record. uri?eid=2-s2.0-53849084810\&partnerID $=40 \& \mathrm{md} 5=3 \mathrm{be} 17 \mathrm{~b} 2 \mathrm{a} 45148387 \mathrm{fd} 08 \mathrm{~b} 91 \mathrm{a}$ ac370748

Pereyra Girardi, C. I., Milei, J., \& Stefani, D. (2011). Factores psicosociales de riesgo en la enfermedad cardiaca. Revista Argentina de Clínica Psicológica, XX(3), 221-229. 
Post Sennehed, C., Gard, G., Holmberg, S., Stigmar, K., Forsbrand, M., \& Grahn, B. (2017). Blue flags", development of a short clinical questionnaire on work-related psychosocial risk factors - A validation study in primary care. BMC Musculoskeletal Disorders, 18(1), 1-11. https://doi. org/10.1186/s12891-017-1677-z

Rashid, M., \& Zimring, C. (2008). A review of the empirical literature on the relationships between indoor environment and stress in health care and office settings - Problems and prospects of sharing evidence. Environment and Behavior, 40(2), 151-190. https://doi. org/10.1177/0013916507311550

Rick, J., Briner, R. B., Daniels, K., Perryman, S., \& Guppy, A. (2001). A Critical Review of Psychosocial Hazard Measure, 148.

Rugulies, R., Aust, B., \& Madsen, I. E. (2017). Effort-reward imbalance at work and risk of depressive disorders. A systematic review and meta-analysis of prospective cohort studies. Scandinavian Journal of Work, Environment \& Health, 43(4), 294-306. https://doi.org/10.5271/sjweh.3632

Schnall, P. L., Dobson, M., \& Landsbergis, P. (2016). Globalization, Work, and Cardiovascular Disease. International Journal of Health Services, 46(4), 656-692. https://doi.org/10.1177/0020731416664687

Shaw, W. S., van der Windt, D. A., Main, C. J., Loisel, P., \& Linton, S. J. (2009). Early Patient Screening and Intervention to Address Individual-Level Occupational Factors $(\{"\}$ Blue Flags $\{"\}$ ) in Back Disability. Journal of Occupational Rehabilitation, 19(1), 64-80. https://doi.org/10.1007/s10926-008-9159-7

Siegrist, J. (2011). Social determinants of health - Contributions from European health and medical sociology. Politica y Sociedad, 48(2). https://doi.org/10.5209/rev-POSO.2011. v48.n2.2
Silva, C., Barros, C., Cunha, L., Carnide, F., \& Santos, M. (2014). Prevalence of back pain problems in relation to occupational group. International Journal of Industrial Ergonomics, 52. https://doi.org/10.1016/j. ergon.2015.08.005

Singh, H., \& Conroy, D. E. (2017). Systematic review of stress-related injury vulnerability in athletic and occupational contexts. Psychology of Sport and Exercise, 33, 37-44. https://doi.org/10.1016/j. psychsport.2017.08.001

Skogstad, A., Torsheim, T., Einarsen, S., \& Hauge, L. J. (2011). Testing the work environment hypothesis of bullying on a group level of analysis: Psychosocial factors as precursors of observed workplace bullying. Applied Psychology, 60(3), 475-495. https://doi. org/10.1111/j.1464-0597.2011.00444.X

Solovieva, S., Lallukka, T., Virtanen, M., \& Viikari-Juntura, E. (2013). Psychosocial factors at work, long work hours, and obesity: A systematic review. Scandinavian Journal of Work, Environment and Health, 39(3). https://doi.org/10.5271/sjweh.3364

Szerencsi, K., van Amelsvoort, L. G. P. M., Viechtbauer, W., Mohren, D. C. L., Prins, M. H., \& Kant, I. J. (2012). The association between study characteristics and outcome in the relation between job stress and cardiovascular disease - A multilevel metaregression analysis. Scandinavian Journal of Work, Environment and Health, 38(6). https://doi.org/10.5271/sjweh.3283

Taylor, J. B., Goode, A. P., George, S. Z., \& Cook, C. E. (2014). Incidence and risk factors for first-time incident low back pain: A systematic review and meta-analysis. Spine Journal, 14(10). https://doi.org/10.1016/j. spinee.2014.01.026 
Thayer, J. F., Yamamoto, S. S., \& Brosschot, J. F. (2010). The relationship of autonomic imbalance, heart rate variability and cardiovascular disease risk factors. International Journal of Cardiology, 141(2). https://doi.org/10.1016/j.ijcard.2009.09.543

Turgoose, D., \& Maddox, L. (2017). Predictors of compassion fatigue in mental health professionals: A narrative review. Traumatology, 23(2), 172-185. https://doi. org/10.1037/trm0000116

Van Laethem, M., Beckers, D. G., Kompier, M. A., Dijksterhuis, A., \& Geurts, S. A. (2013). Psychosocial work characteristics and sleep quality: a systematic review of longitudinal and intervention research. Scandinavian Journal of Work, Environment \& Health, 39(6), 535-549. https://doi.org/10.5271/ sjweh.3376

Villalobos, G. H., Vargas, A. M., Rondón, M. A., \& Felknor, S. A. (2013). Design of psychosocial factors questionnaires: A systematic measurement approach. American Journal of Industrial Medicine, 56(1), 100110. https://doi.org/10.1002/ajim.22071

Violante, F. S., Mattioli, S., \& Bonfiglioli, R. (2015). Low-back pain. Handbook of Clinical Neurology. Occupational Health Unit, Department of Medical and Surgical Sciences, University of Bologna, Bologna, Italy: Elsevier. https://oi.org/10.1016/ B978-0-444-62627-1.00020-2
Vischer, J. C. (2007). The effects of the physical environment on job performance: Towards a theoretical model of workspace stress. Stress and Health, 23(3). https://doi.org/10.1002/ smi. 1134

Waters, T., Genaidy, A., Viruet, H. B., \& Makola, M. (2008). The impact of operating heavy equipment vehicles on lower back disorders. Ergonomics, 51(5), 602-636. https://doi. org/10.1080/00140130701779197

Weissbrodt, R., \& Giauque, D. (2017). Labour inspections and the prevention of psychosocial risks at work: A realist synthesis. Safety Science, 100, 110-124. https://doi.org/10.1016/j.ssci.2017.02.012

Zare, M., Croq, M., Hossein-Arabi, F., Brunet, R., \& Roquelaure, Y. (2016). Does Ergonomics Improve Product Quality and Reduce Costs? A Review Article. Human Factors and Ergonomics In Manufacturing \& Service Industries, 26(2), 205-223. https://doi. org/10.1002/hfm.20623

Zoni, S., \& Lucchini, R. G. (2012). European approaches to work-related stress: A critical review on risk evaluation. Safety and Health at Work, 3(1). https://doi.org/10.5491/ SHAW.2012.3.1.43 